\title{
Performance of MAP Channel Estimator in Power Line Communication with OFDM under Impulsive Noise
}

\author{
S. Muratoğlu Çürük
}

\begin{abstract}
Power Line Communication (PLC), which is a competitive technique especially for in-home applications, faces impulsive noise together with unstable channel characteristics. These two unpredictable factors make the communication on power lines difficult, which may reduce the efficiency of Orthogonal Frequency Division Multiplexing (OFDM), the selected modulation standard for PLC. There exists various channel estimators for PLC in the literature. The former techniques assume the channel noise is white Gaussian as in the wireless multipath, while the newer ones model the corrupted noise as impulsive which results with more complex receivers. In this study, the performance of the Maximum A-Posteriori (MAP) estimator proposed for additive white Gaussian noise channels is evaluated for PLC systems with OFDM under the constraint of impulsive noise. For the simulations multipath channel data is taken from the literature and Middleton Class A impulsive noise model is used. The results show that the MAP estimator which is designed with the assumption of white Gaussian noise has a satisfactory performance even under heavy impulsive noise.
\end{abstract}

Index Terms - Channel estimation, impulsive noise, Orthogonal Frequency Division Multiplexing, Power Line Communication.

\section{INTRODUCTION}

In recent years, studies related to the performance of the popular technique, Power Line Communication (PLC) is increasing rapidly. The main superiority of PLC is that it does not request any specific wiring for communication because it uses the already existing power line network. For communication, though they are wired, power line channels have multipath characteristics like the wireless channels and the parameters of the multipath channels vary with different network topologies and loads. Moreover, PLC may be damaged by disturbances, such as narrowband interference and additive, white/colored and/or impulsive noise. Unfortunately, all these factors result with unstable varying channel characteristics, which is the primary restricting factor for the data rate of PLC modems that has to struggle with alternatives.

S. M. ÇÜRÜK, is with Department of Electrical and Electronics Engineering University of Iskenderun Technical University, Hatay, Turkey, (e-mail: selva.curuk@iste.edu.tr)(iD

Manuscript received September 18, 2017; accepted January 9, 2018. DOI: $10.17694 /$ bajece. 401998
For a success power line networks should be considered carefully and this increases the complexity of PLC systems. The multicarrier modulation technique named Orthogonal Frequency Division Multiplexing (OFDM) overcomes multipath fading effects by dividing the wide band into many narrower subbands where it uses orthogonal carriers for bandwidth efficiency. OFDM has been popular for wireless communication systems and has also been selected by Home Plug Powerline Alliance as power line modulation standard [1]. The performance of an OFDM system is highly dependent on the quality of the channel estimation. Typically, in OFDM systems subchannel coefficients are highly correlated. Thus the Maximum A-Posteriori (MAP) based estimation techniques are expected to perform better than the Maximum Likelihood (ML) based ones: The ML estimates will have a higher Mean Square Error (MSE) especially for low Signal to Noise Ratios (SNR).

Although the number is limited, channel estimators for OFDM systems in PLCs have been proposed in the literature. The former techniques assume the channel noise is white Gaussian as in the wireless multipath, while the newer ones are more realistic and model the corrupted noise as impulsive which results with more complex receiver structures. Further, adaptive estimator structures proposed, which estimate the channel and noise dynamically, are declared to have better performance but even more complicated receivers. For example in [2], to reduce impulsive noise, an algorithm for channel estimation is given basing on a robust cost function. In [3], a discrete-time parametric representation of a linear periodically time-varying system is adopted to model indoor power line channels. Following, the parameters of the proposed representation are estimated. In [4], the robust relevance vector machine based channel estimation is used. The authors in [5] present an adaptive iterative receiver used to decrease the impact of impulsive noise on OFDM based power line communications. In [6], power line channel impulse response estimation is done by applying a relevance vector machine to the received data. The authors of [7] cancel the impulsive noise in PLC systems with OFDM by steps settled on turbo coding and frequency domain equalization. The adaptive algorithm in [8] for impulsive noise suppression seen over the PLC channel applies an iterative estimation. In [9], regardless of the statistics of the noise, the authors give the equivalence of Minimum SNR estimator and Minimum MSE estimator. In [10], authors have used the Generalized Eigenvalues Utilizing Signal Subspace 
Eigenvectors algorithm for multipath parameter estimation of PLC channel. The authors of [11] provide an iterative impulsive noise reduction technique based on MMSE/MAP estimation for OFDM communications.

Before dealing with complicated receivers, the question that should be answered is that, do we really need such complex receivers in practice? What is the degradation in performance if we use simple estimation techniques? Therefore, in this study, we investigate the degradation in performance if we use the MAP estimator proposed for additive white Gaussian noise channels for PLC systems with OFDM under the constraint of impulsive noise.

\section{POWER LINE CHANNELS}

PLC channels have frequency selective and time varying multipath behavior, depending on the network topology and wire type. The studies have shown that a PLC channel may be modeled as a multipath channel, and its frequency response is dedicated by [12]

$$
H(f, t)=\sum_{i=1}^{L_{p}} g_{i, t} \cdot e^{-\left(a_{0}+a_{1} f^{k}\right) d_{i}} \cdot e^{-j 2 \pi f\left(d_{i} / v_{p}\right)},
$$

where $g_{i, t}$ is the time dependent weighting factor of $i$-th path. $e^{-\left(a_{0}+a_{1} f^{k}\right) d_{i}}$ is the attenuation part $\left(a_{0}\right.$ and $a_{1}$ are attenuation parameters, $k$ is the exponent of the attenuation factor, $d_{i}$ is the length of $i$-th path) and $e^{-j 2 \pi f\left(d i / v_{p}\right)}$ is the delay portion $\left(v_{p}\right.$ is the propagation velocity).

In this study, three different PLC multipath channel model taken from the literature are used: $\mathrm{CH} 1$, is a 4-path multipath channel which is suitable for PLC channels with 1-4 branch network topology and short distance in the range of $100-200$ $\mathrm{m}$ [12]. The attenuation parameters are $a_{0}=0, a_{1}=7.8 \cdot 10^{-10}$, $k=1$, and the propagation velocity $v_{p}=1.5 \cdot 10^{8}$. $\mathrm{CH} 2$ is again for short distance channels with an 8-path multipath, but with different attenuation parameters: $a_{0}=0, a_{1}=1 \cdot 10^{-7}, k=0.6$ [13]. CH3 has 15 path, and is a model for longer distance, longer than $300 \mathrm{~m}$, with a more complicated network topology [14]. The parameters are $a_{0}=0, a_{1}=7.8 \cdot 10^{-10}, k=1$, and $v_{p}=1.5 \cdot 10^{8}$. The path weighting factor $g_{i, t}$ and path length $d_{i}$ , for $\mathrm{CH} 1, \mathrm{CH} 2$ and $\mathrm{CH} 3$ are inscribed in Table 1, Table 2 and Table 3, respectively.

TABLE I

CHANNEL I (CH1) PARAMETERS

\begin{tabular}{|c|c|c|c|c|}
\hline Path no & $\mathbf{1}$ & $\mathbf{2}$ & $\mathbf{3}$ & $\mathbf{4}$ \\
\hline$g_{i, t}$ & 0.64 & 0.38 & -0.15 & 0.05 \\
\hline$d_{i}(\mathrm{~m})$ & 200 & 222.4 & 244.8 & 267.5 \\
\hline
\end{tabular}

TABLE II CHANNEL II (CH2) PARAMETERS

\begin{tabular}{|c|c|c|c|c|}
\hline Path no & $\mathbf{1}$ & $\mathbf{2}$ & $\mathbf{3}$ & $\mathbf{4}$ \\
\hline$g_{i, t}$ & 0.6 & 0.11 & -0.12 & 0.11 \\
\hline$d_{i}(\mathrm{~m})$ & 15 & 22 & 28 & 35 \\
\hline Path no & $\mathbf{5}$ & $\mathbf{6}$ & $\mathbf{7}$ & $\mathbf{8}$ \\
\hline$g_{i, t}$ & 0.07 & -0.07 & 0.06 & -0.01 \\
\hline$d_{i}(\mathrm{~m})$ & 41 & 48 & 53 & 59 \\
\hline
\end{tabular}

TABLE III CHANNEL III (CH3) PARAMETERS

\begin{tabular}{|c|c|c|c|c|c|}
\hline Path no & $\mathbf{1}$ & $\mathbf{2}$ & $\mathbf{3}$ & $\mathbf{4}$ & $\mathbf{5}$ \\
\hline$g_{i, t}$ & 0.029 & 0.043 & 0.103 & -0.058 & -0.045 \\
\hline$d_{i}(\mathrm{~m})$ & 75 & 85 & 94.2 & 119.2 & 123.3 \\
\hline Path no & $\mathbf{6}$ & $\mathbf{7}$ & $\mathbf{8}$ & $\mathbf{9}$ & $\mathbf{1 0}$ \\
\hline$g_{i, t}$ & -0.040 & 0.038 & -0.038 & 0.071 & -0.035 \\
\hline$d_{i}(\mathrm{~m})$ & 166.7 & 216.7 & 268.3 & 342.5 & 408.3 \\
\hline Path no & $\mathbf{1 1}$ & $\mathbf{1 2}$ & $\mathbf{1 3}$ & $\mathbf{1 4}$ & $\mathbf{1 5}$ \\
\hline$g_{i, t}$ & 0.065 & -0.055 & 0.042 & -0.059 & 0.049 \\
\hline$d_{i}(\mathrm{~m})$ & 472.5 & 616.7 & 800 & 941.7 & 1041.7 \\
\hline
\end{tabular}

The noise sources observed in PLC channels may be grouped according to their durations. The first group is stationary over a long period compared to symbol duration and is called background noise. It is a combination of colored background noise, narrowband noise and periodic impulsive noise asynchronous to the mains frequency. The second group is time variant with short durations of microseconds to milliseconds. This group is a combination of asynchronous impulsive noise and periodic impulsive noise synchronous to the mains frequency. Time domain impulsive noise in PLC is modelled frequently with Middleton's Class A noise model. The probability density function (pdf) of a Middleton Class A noise sample $n_{k}$ is given by [15]:

$$
p\left(n_{k}\right)=\sum_{m=0}^{\infty} P_{m} \aleph\left(n_{k} ; 0, \sigma_{m}^{2}\right),
$$

where the mixing probability $P_{m}$ is given by

$$
P_{m}=\frac{A^{m} \cdot e^{-A}}{m !} .
$$

The "impulsive index" A gives the density of impulses in an observation period. It should be specified that $A \in\left[10^{-2}, 1\right]$ for PLC channels. $\aleph\left(n_{k} ; \mu, \sigma_{m}{ }^{2}\right)$ is the Gaussian pdf with mean $\mu$ and variance $\sigma_{m}{ }^{2}$. The variance in Middleton Class A model is defined as

$$
\sigma_{m}^{2}=\sigma_{I}^{2} \cdot \frac{m}{A}+\sigma_{g}^{2}
$$

or equivalently, 


$$
\sigma_{m}{ }^{2}=\sigma_{g}{ }^{2} \cdot\left(\frac{m}{A \cdot \Gamma}+1\right)
$$

Here $\sigma_{I}^{2}$ and $\sigma_{g}{ }^{2}$ are the variances of the impulse noise and the background Gaussian noise, respectively. The Gaussian factor $\Gamma=\sigma_{g}^{2} / \sigma_{I}^{2}$ is the ratio of Gaussian to impulse noise power. The Gaussian factor is generally in the range $\left[10^{-6}, 1\right]$.

\section{ChANNEL EstimatorS}

The PLC channel is assumed to be stationary inside the observation interval. Further, the channel band is frequency selective, but flat for subbands of OFDM. Then, after removing the modulation effects, the received vector for an OFDM system with $N$ subcarriers is:

$$
\mathbf{r}=\mathbf{h}+\mathbf{n},
$$

where the additive noise $\mathbf{n}$ is impulsive and the channel coefficients $\mathbf{h}$ are samples of zero mean jointly Gaussian complex random process with covariance matrix $\mathbf{C}_{\mathbf{h}}$. The MAP estimation of the channel coefficient vector [16] is found by:

$$
\hat{\mathbf{h}}_{\mathbf{M A P}}=\underset{\mathbf{z}}{\arg \max } p(\mathbf{h} / \mathbf{r})
$$

If the impulsive noise is modeled as zero mean white Gaussian with covariance matrix $\mathbf{C}_{\mathbf{n}}$, then

$$
\begin{gathered}
p(\mathbf{r} / \mathbf{h})=\frac{1}{\pi^{N} \cdot\left|\mathbf{C}_{\mathbf{n}}\right|} \exp \left[-(\mathbf{r}-\mathbf{h})^{\mathbf{H}} \cdot \mathbf{C}_{\mathbf{n}}{ }^{-\mathbf{1}} \cdot(\mathbf{r}-\mathbf{h})\right] \\
p(\mathbf{h})=\frac{1}{\pi^{N} \cdot\left|\mathbf{C}_{\mathbf{h}}\right|} \exp \left[-\mathbf{h}^{\mathbf{H}} \cdot \mathbf{C}_{\mathbf{z}}{ }^{-\mathbf{1}} \cdot \mathbf{h}\right]
\end{gathered}
$$

Following necessary steps it can be shown that the MAP estimates are given by:

$$
\hat{\mathrm{h}}_{\mathrm{MAP}}=\left(\mathrm{C}_{\mathrm{n}}{ }^{-1}+\mathrm{C}_{\mathrm{h}}{ }^{-1}\right)^{-1} \cdot \mathrm{C}_{\mathrm{n}}{ }^{-1} \cdot \mathbf{r}
$$

and simplifying (10), MAP estimation expression is found to be

$$
\hat{\mathbf{h}}_{\mathbf{M A P}}=\mathbf{B} \cdot \mathbf{r} \quad \text { where } \quad \mathbf{B}=\mathbf{C}_{\mathbf{h}} \cdot\left(\mathbf{C}_{\mathbf{n}}+\mathbf{C}_{\mathbf{h}}\right)^{-\mathbf{1}}
$$

The MSE matrix for the MAP estimates is given by [16]:

$$
\operatorname{MSE}_{\text {MAP }}=\mathbf{B} \cdot \mathbf{C}_{\mathbf{n}} \cdot \mathbf{B}^{\mathbf{H}}+\left(\mathrm{B}-\mathbf{I}_{\mathbf{N}}\right) \cdot \mathbf{C}_{\mathbf{h}} \cdot\left(\mathrm{B}-\mathbf{I}_{\mathbf{N}}\right)^{\mathbf{H}}
$$

where $\mathbf{I}_{\mathbf{N}}$ is the $N \mathbf{x} N$ identity matrix and $(.)^{\mathrm{H}}$ means Hermitian of the matrix. Equivalently, it can be written as

$$
\operatorname{MSE}_{\text {MAP }}=C_{h}-C_{h} \cdot\left(C_{n}+C_{h}\right)^{-1} \cdot C_{h}
$$

Note that, under the constraint of white noise, the noise samples are mutually independent, i.e., $\mathbf{C}_{\mathbf{n}}=\sigma_{n}^{2} . \mathbf{I}_{\mathbf{N}}$, where $\sigma_{n}^{2}$ is white noise variance, then (13) simplifies to

$$
\mathrm{MSE}_{\mathbf{M A P}}=\sigma_{n}^{2} \cdot \mathbf{B}
$$

For ML estimation (without a priori knowledge of the channel), B will be the identity matrix. Then the ML estimate [16] is $\hat{\mathbf{h}}_{\mathbf{M L}}=\mathbf{r}$, and its MSE is $\mathbf{M S E}_{\mathbf{M L}}=\mathbf{C}_{\mathbf{n}}$.

\section{Simulation Results}

For the simulations, we have taken the HomePlug AV standard [1] as reference. The standard states that OFDM with 1155 subcarrier is used as modulation scheme in the band 1.8 to $30 \mathrm{MHz}$. Therefore in the simulations $0-30 \mathrm{MHz}$ band is considered (elementary period $T$ is $1 / 30 \mu \mathrm{s}$ ) and the subcarrier number, $N$, is selected to be 1200 ( $25 \mathrm{kHz}$ subbands).

Fig. 1, Fig. 2 and Fig. 3 depict multipath characteristics of PLC channels that will be estimated, whose parameters are given in Section II. The amplitudes are determined from the samples taken from the amplitude spectra of the channels where $n$ is the subchannel indices. As seen from the figures the frequency selectivity is lowest for $\mathrm{CH} 2$. The amplitude attenuation that exists in $\mathrm{CH} 1$ rapidly increases with frequency. And for $\mathrm{CH} 3$, there are sudden changes in the multipath characteristics in 0-200 subbands (0-5 MHz band), which is more serious in 0-100 subbands (0-2.5 MHz band).

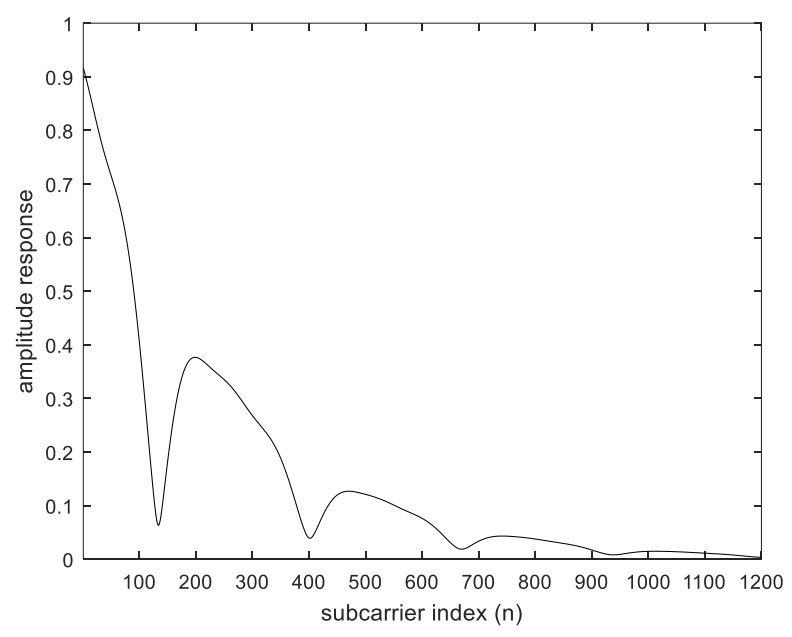

Fig. 1. Amplitude response of $\mathrm{CH} 1$ versus subcarrier index. 


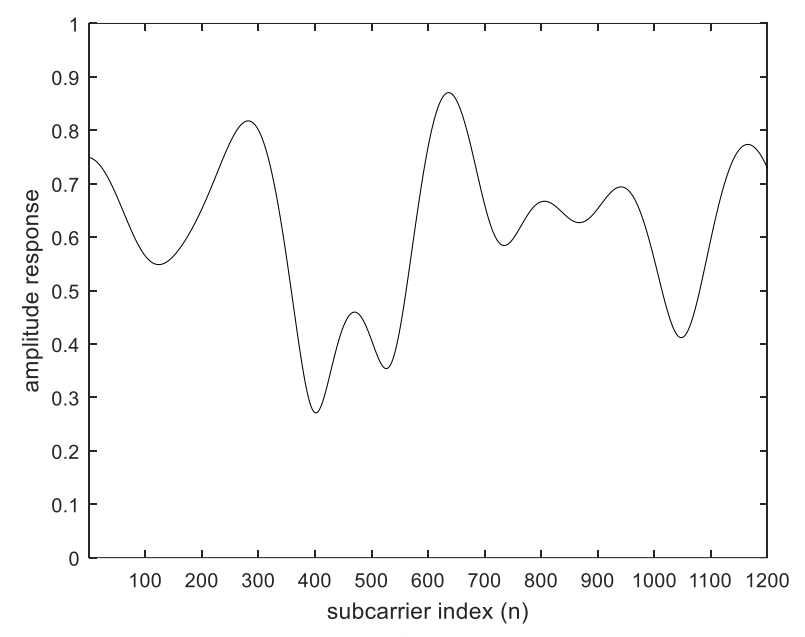

Fig. 2. Amplitude response of $\mathrm{CH} 2$ versus subcarrier index

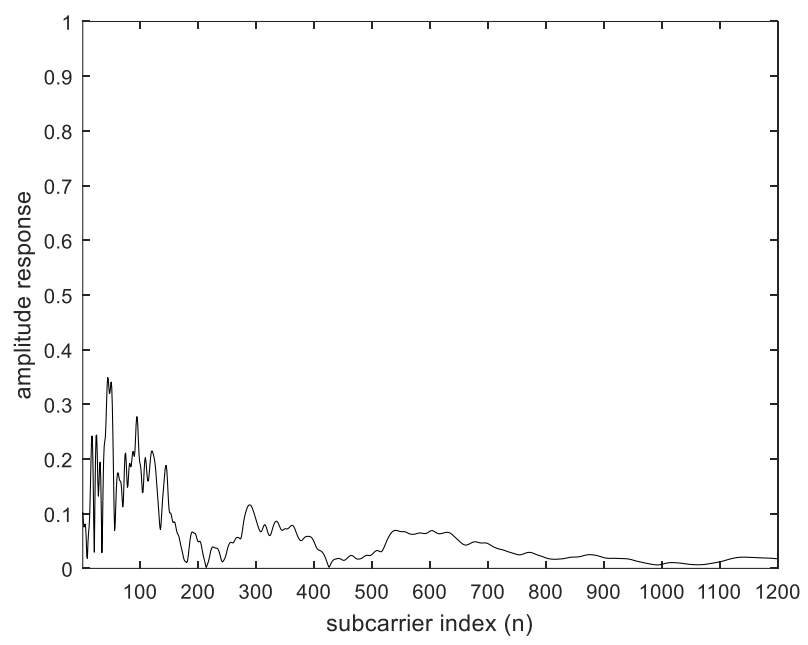

Fig. 3. Amplitude response of $\mathrm{CH} 3$ versus subcarrier index.

Simulation results of MAP channel estimator rms errors with Gaussian noise versus subchannel number for $\mathrm{CH} 1, \mathrm{CH} 2$ and $\mathrm{CH} 3$ are given in Fig. 4, Fig. 5, and Fig. 6, respectively. Simulation parameters are selected to be SNR $=10 \mathrm{~dB}, N=1200$. The performance of the MAP estimator with Gaussian noise assumption is also analyzed under the Middleton Class A model impulsive noise with parameter values are selected to be $A=1, \Gamma=10^{-6}$ (highly impulsive case) with same SNR and subcarrier number as in the previous simulations. Simulation results for $\mathrm{CH} 1, \mathrm{CH} 2$ and $\mathrm{CH} 3$ are again given in Fig. 4, Fig. 5, and Fig. 6, respectively. As seen, the performance of the MAP estimator designed for Gaussian noise is good under the constraint of impulsive noise for all channels, as in white noise case. The average MSEs under Gaussian noise are $0.0022,0.0024$ and 0.0003 for $\mathrm{CH} 1, \mathrm{CH} 2$ and $\mathrm{CH} 3$, respectively. Under the impulsive noise, the averages are 0.0024, 0.0028 and 0.0003 for $\mathrm{CH} 1, \mathrm{CH} 2$ and $\mathrm{CH} 3$, respectively. MAP estimator designed with the assumption of Gaussian noise performs well in all channels even in the worst case of impulsive noise when SNR is $10 \mathrm{~dB}$.
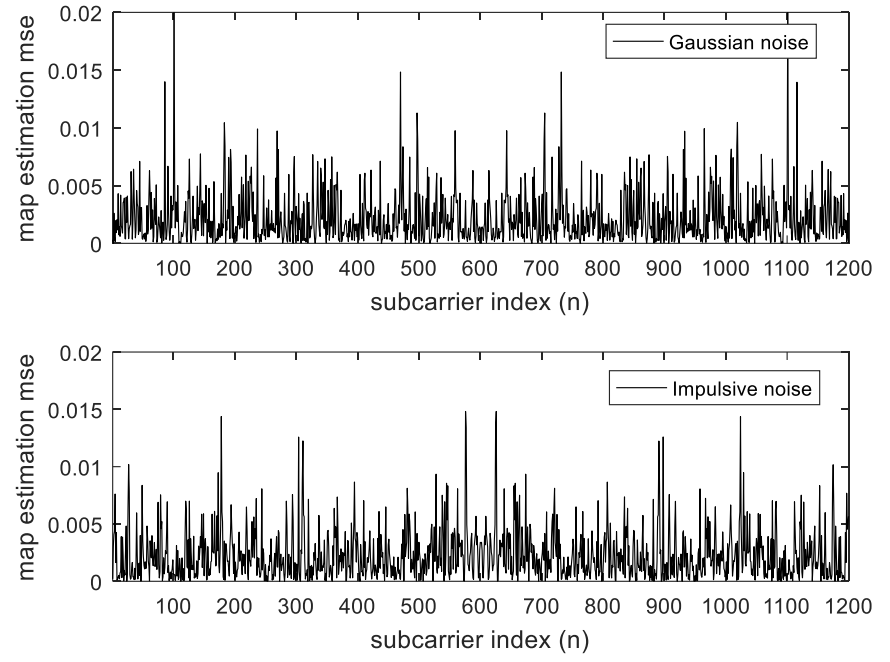

Fig. 4. MAP channel estimator rms error, $\mathrm{SNR}=10 \mathrm{~dB}, \mathrm{CH} 1$, Gaussian and Impulsive noise.
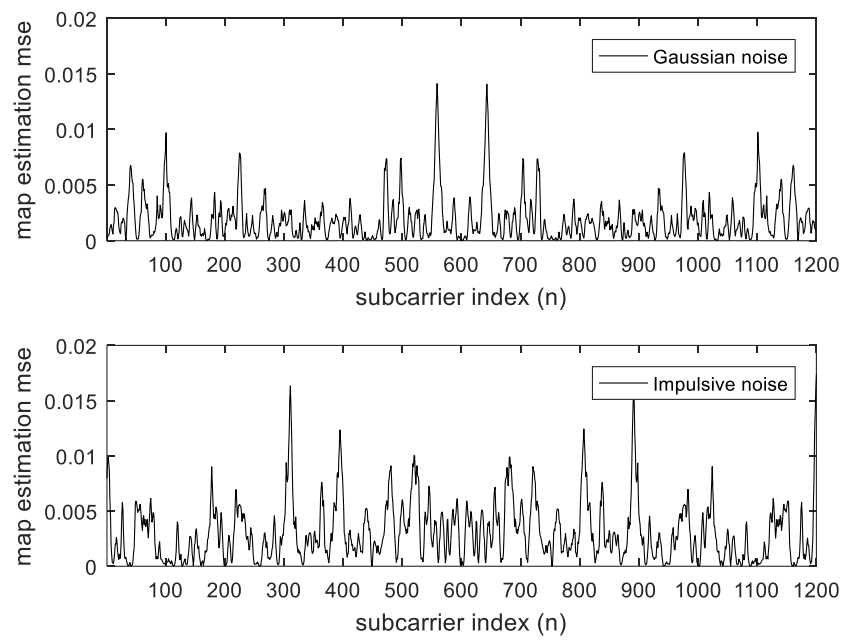

Fig. 5. MAP channel estimator rms error, $\mathrm{SNR}=10 \mathrm{~dB}, \mathrm{CH} 2$, Gaussian and Impulsive noise.
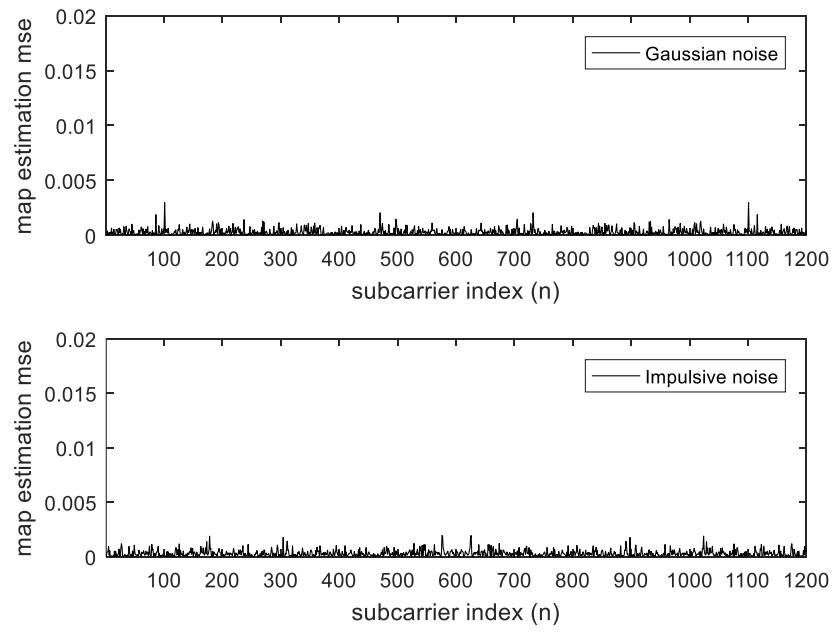

Fig. 6. MAP channel estimator rms error, SNR $=10 \mathrm{~dB}, \mathrm{CH} 3$, Gaussian and Impulsive noise. 
The simulations are repeated for $\mathrm{SNR}=0 \mathrm{~dB}$ to investigate the performance for low SNR case. The results are given below in Fig. 7, Fig. 8 and Fig. 9. The average MSEs of estimator under Gaussian noise are found to be $0.0059,0.0092$ and 0.0012 for $\mathrm{CH} 1, \mathrm{CH} 2$ and $\mathrm{CH} 3$, respectively. The average MSEs of estimator under impulsive noise are $0.0064,0.0106$ and 0.0014 for $\mathrm{CH} 1, \mathrm{CH} 2$ and $\mathrm{CH} 3$, respectively. Again we concluded that although there is a slight degradation in performance, the MAP estimator designed with the assumption of Gaussian noise performs well in all channels.

The simulation results showed that all performances are very close to the case of channel with Gaussian noise even in the worst case of impulsive noise and low SNR. This is because of the FFT operation in OFDM receiver. OFDM systems randomized the power line impulsive noise via its FFT operation, resulting in Gaussian distributed noise, especially if the number of OFDM subcarriers is sufficiently large.
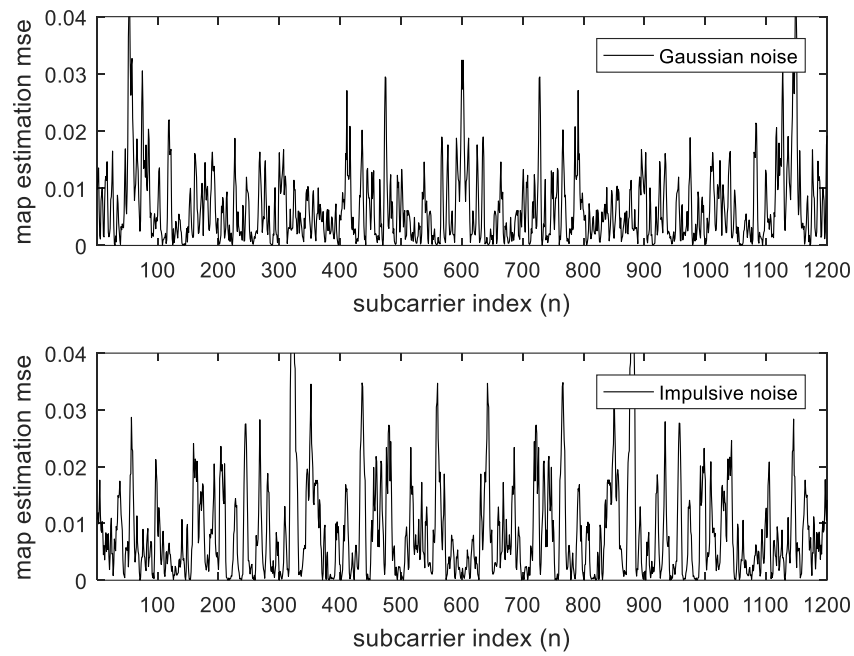

Fig. 7. MAP channel estimator rms error, $\mathrm{SNR}=0 \mathrm{~dB}, \mathrm{CH} 1$, Gaussian and Impulsive noise.
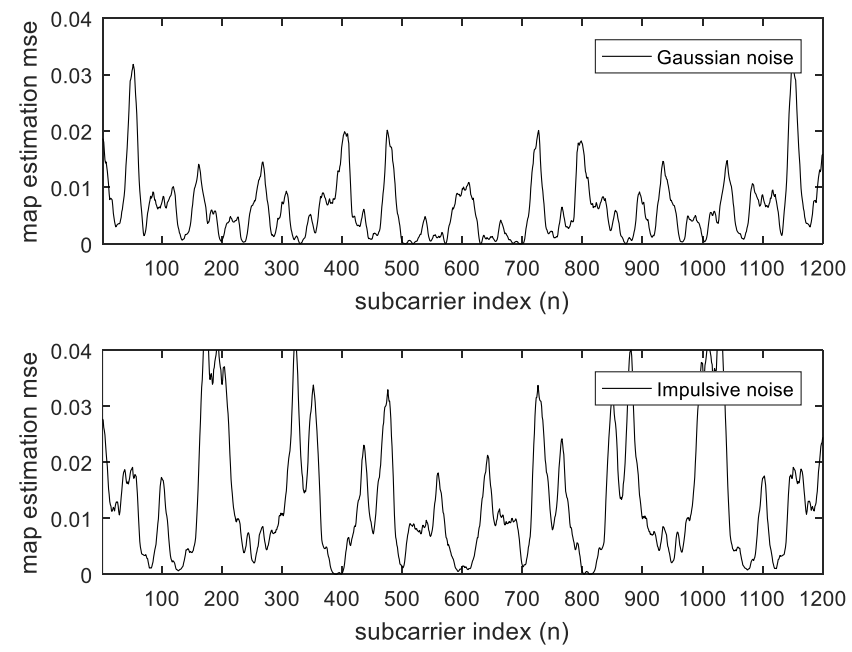

Fig. 8. MAP channel estimator rms error, $\mathrm{SNR}=0 \mathrm{~dB}, \mathrm{CH} 2$, Gaussian and Impulsive noise.
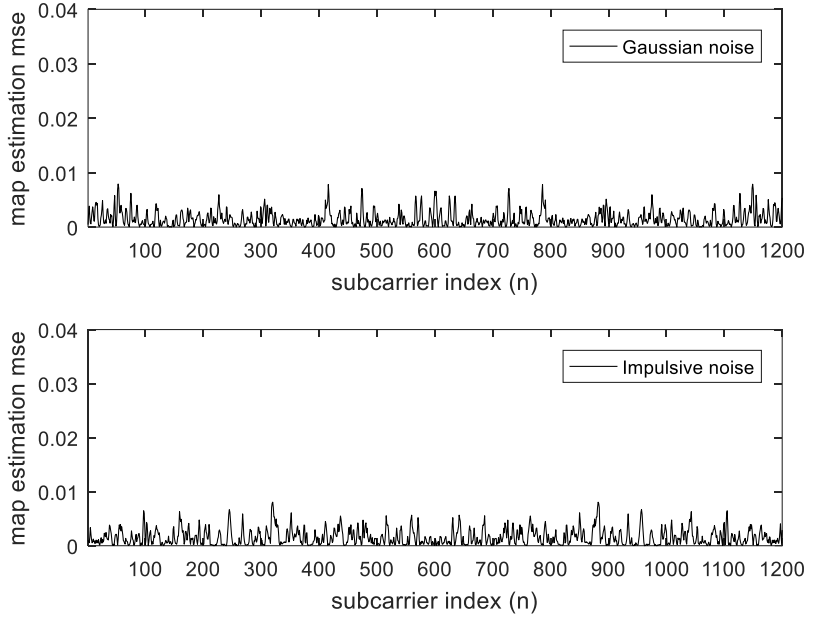

Fig. 9. MAP channel estimator rms error, $\mathrm{SNR}=0 \mathrm{~dB}, \mathrm{CH} 3$, Gaussian and Impulsive noise.

\section{CONCLUSION}

In this study, we aimed to analyze the degradation in the performance of MAP channel estimator if we ignore the noise is impulsive and assume it Gaussian. Thus, the performance of MAP channel estimator proposed for additive white Gaussian noise channels is evaluated for PLC systems with OFDM under the constraint of impulsive noise. The simulations are done using three different channel data taken from the literature and Middleton Class A impulsive noise model. The MSE of estimations under heavy impulsive noise circumstance is investigated. The results show that the degradation in performance is insignificant, only distinctive for low SNR, for all channels. The MAP estimator which is designed for white Gaussian noise has a quite satisfactory performance even under heavy impulsive noise and can be safely used. The reason is that in an OFDM system, the impulsive noise is randomized via the FFT operation, thus noise is spread over all carrier frequencies and behaves like Gaussian even if it is impulsive in time domain. We conclude that we can safely assume the noise to be Gaussian in frequency domain

\section{REFERENCES}

[1] HomePlug Powerline Alliance Inc. (2005). "HomePlug AV White Paper", Available: http://www.homeplug.org/media/filer_public/b8/ 68/b86828d9-7e8a-486f-aa82-179e6e95cab5/hpav-whitepaper_050818.pdf, date on 17.05.2017.

[2] J. Huang, P. Wang, Q. Wan, "Robust approach for channel estimation in power line communication", Journal of Communications and Networks, Vol.14, No.3, 2012, pp.237-242.

[3] F. Gianaroli, F. Pancaldi, G.M. Vitetta, "On the use of Zadeh's series expansion for modeling and estimation of indoor powerline channels", IEEE Transactions on Communications, Vol.62, No.7, 2014, pp.25582568.

[4] M. Asadpour, F.A. Ajhiri, B.M. Tazehkand, et al., "Jointly RVM based channel estimation and PAPR reduction using Modified Tabu Search Algorithm in Power Line Communication systems", Wireless Personal Communications, Vol.84, No.4, 2015, pp.2757-2775.

[5] Y.R. Chien, "Iterative channel estimation and impulsive noise mitigation algorithm for OFDM-based receivers with application to Power-line communications", IEEE Transactions on Power Delivery, Vol.30, No.6, 2015, pp.2435-2442. 
[6] M. Asadpour, B.M. Tazehkand, H. Seyedarabi, "A robust Bayesian inference-based channel estimation in power line communication systems contaminated by impulsive noise", Turkish Journal of Electrical Engineering and Computer Sciences, Vol.24, No.4, 2016, pp.2865-2880.

[7] Y. Himeur, A. Boukabou, A. Senouci, "Performance of turbo-coded chaotic interleaving and frequency-domain equalization scheme for high speed OFDM-based PLC systems", Journal of the Franklin Institute, Vol.353, No.15, 2016, pp.3799-3817.

[8] Y. Himeur, A. Boukabou, "OFDM-based power-line communication enhancement using a turbo coded adaptive impulsive noise compensator", Telecommunication Systems, Vol.62, No.3, 2016, pp.481494.

[9] L. Rugini, P. Banelli, "On the equivalence of Maximum SNR and MMSE estimation: Applications to additive non-gaussian channels and quantized observations", IEEE Transactions on Signal Processing, Vol.64, No.23, 2016, pp. 6190-6199.

[10] Y.H. Kim, "Multipath parameter estimation for PLC channels using the GEESE algorithm", IEEE Transactions on Power Delivery, Vol.25, No.4, 2010, pp. 2339-2345.

[11] P.A.C. Lopes, J.A.B. Gerald, "Iterative MMSE/MAP impulsive noise reduction for OFDM", Digital Signal Processing, Vol.69, 2017, pp.252258.

[12] M. Zimmermann, K. Dostert, "A multipath model for the powerline channel", IEEE Transactions on Communication, Vol.50, No.4, 2002, pp.553-559.

[13] X. Ding, J. Meng, B.R. Petersen, "Blind channel estimation for HomePlug power-line communications: A feasibility?", IEEE Transactions on Power Delivery, Vol.26, No.2, 2011, pp. 754-763.

[14] A.A.M. Picorone, L.R. Amado, M.V. Ribeiro, "Linear and periodically time-varying PLC channels estimation in the presence of impulsive noise", IEEE International Symposium on Power Line Communications and Its Applications, 2010, pp.255-260.

[15] T. Shongwe, A.J.H. Vinck, H.C. Ferreira, "A study on impulse noise and its models", SAIEE Africa Research Journal, Vol.106, No.3, 2015, pp.119-131.

[16] S.M. Curuk, Y. Tanik, "A simplified MAP channel estimator for OFDM systems under Rayleigh fading", European Transactions on Telecommunications, Vol.21, No.4, 2010, pp.396-405.

\section{BIOGRAPHIES}

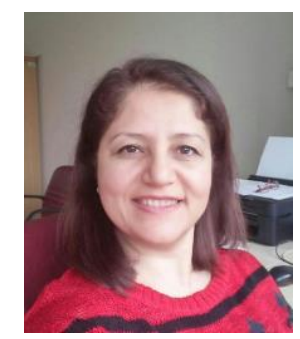

SELVA MURATOĞLU ÇÜRÜK received the B.S. degree from the Electrical and Electronics Engineering Department, Middle East Technical University (METU), Ankara, Turkey, in 1996, and the M.S. and Ph.D. degrees from the same university, in 1999 and 2008, respectively. She is currently an Assistant Professor at the Iskenderun Technical University.

Her research interests are wireless communication systems, digital communication theory, digital signal processing, multicarrier modulation and channel modeling. 